\title{
Multiple stents for tracheobronchomalacia caused by hydrochloric acid inhalation: a case report
}

\author{
Jiwang Wang, Lei Gao, Qi Tan, Wenkui Sun \\ Department of Respiratory and Critical Care Medicine, The First Affiliated Hospital, Nanjing Medical University, Nanjing, China \\ Correspondence to: Wenkui Sun. Department of Respiratory and Critical Care Medicine, The First Affiliated Hospital, Nanjing Medical University, \\ Nanjing 210000, China. Email: sunwenkui@njmu.edu.cn.
}

\begin{abstract}
Tracheobronchomalacia (TBM) is a rare disease characterized by excessive collapsibility of the central airways during expiration. Potential consequences and treatment courses of the aspiration of erosive agents are seldom reported. To our knowledge, this is the first report of TBM caused by hydrochloric acid inhalation, which was successfully treated by multiple airway stents insertion. A 45-year-old female presented with dyspnea and pharynx discomfort after falling into a hydrochloric acid pool. The patient was successfully treated with multiple stents insertion. In 15 months after the stents insertion, the clinical symptoms have been rapidly improved. Inhaled chemical damage may lead to TBM, while multiple airway stents insertion showed a good therapeutic effect for such special TBM.
\end{abstract}

Keywords: Tracheobronchomalacia (TBM); inhalation of dilute hydrochloric acid; airway stent; case report

Submitted Aug 03, 2019. Accepted for publication Jul 28, 2020.

doi: $10.21037 /$ apm-19-216

View this article at: http://dx.doi.org/10.21037/apm-19-216

\section{Introduction}

Tracheobronchomalacia (TBM) refers to the softness of trachea and bronchus which may or may not lead to dynamic airflow limitation. Severe TBM is often a life-threatening condition requiring more aggressive management. The mainstay of therapy for severe TBM was tracheostomy and long-term mechanical ventilation (MV). Internal tracheal stent placement with a silicone prosthesis was first attempted in 1965 by Montgomery (1). Stent placement in patients with TBM is attracting more attention. We haven't found any case of TBM caused by hydrochloric acid inhalation. Here, we present a rare case of patient with TBM who first presented with dyspnea due to hydrochloric inspiration. And we tried a special therapeutic method with multiple stents insertion. Moreover, the case gains better outcomes in a long follow-up. The details of this case are as follows. We present the following case in accordance with the CARE reporting checklist (available at http://dx.doi.org/10.21037/apm-19-216).

\section{Case presentation}

A 45-year-old female presented with dyspnea for 7 weeks and aggravating for 4 days. Seven weeks beforehand, she experienced dyspnea after falling into a hydrochloric acid pool (concentration, 20\%) for 1 minute (Figure 1). She had no special past medical history. Family history was unremarkable. In local hospital, arterial blood gas (ABG) analysis revealed respiratory failure type I. Tracheotomy and MV were promoted subsequently. Bronchoscopy revealed many pseudomembranes and sporadic mucosal erosion.

Seven weeks later, she was transferred to our hospital. When she was treated with MV (oxygen concentration, $100 \%)$, the airway pressure increased and oxygen saturation decreased. ABG analysis results were: $\mathrm{pH}$ 7.107, $\mathrm{PCO}_{2}$ 112.6 and $\mathrm{PO}_{2} 162 \mathrm{mmHg}$. An emergent respiratory interventional therapy was performed. Bronchoscopy evidenced obvious subtotal stenosis (about $80 \%$ ) in the middle and upper parts of trachea. The airway stenosis was considered to be caused by the softness of trachea and 


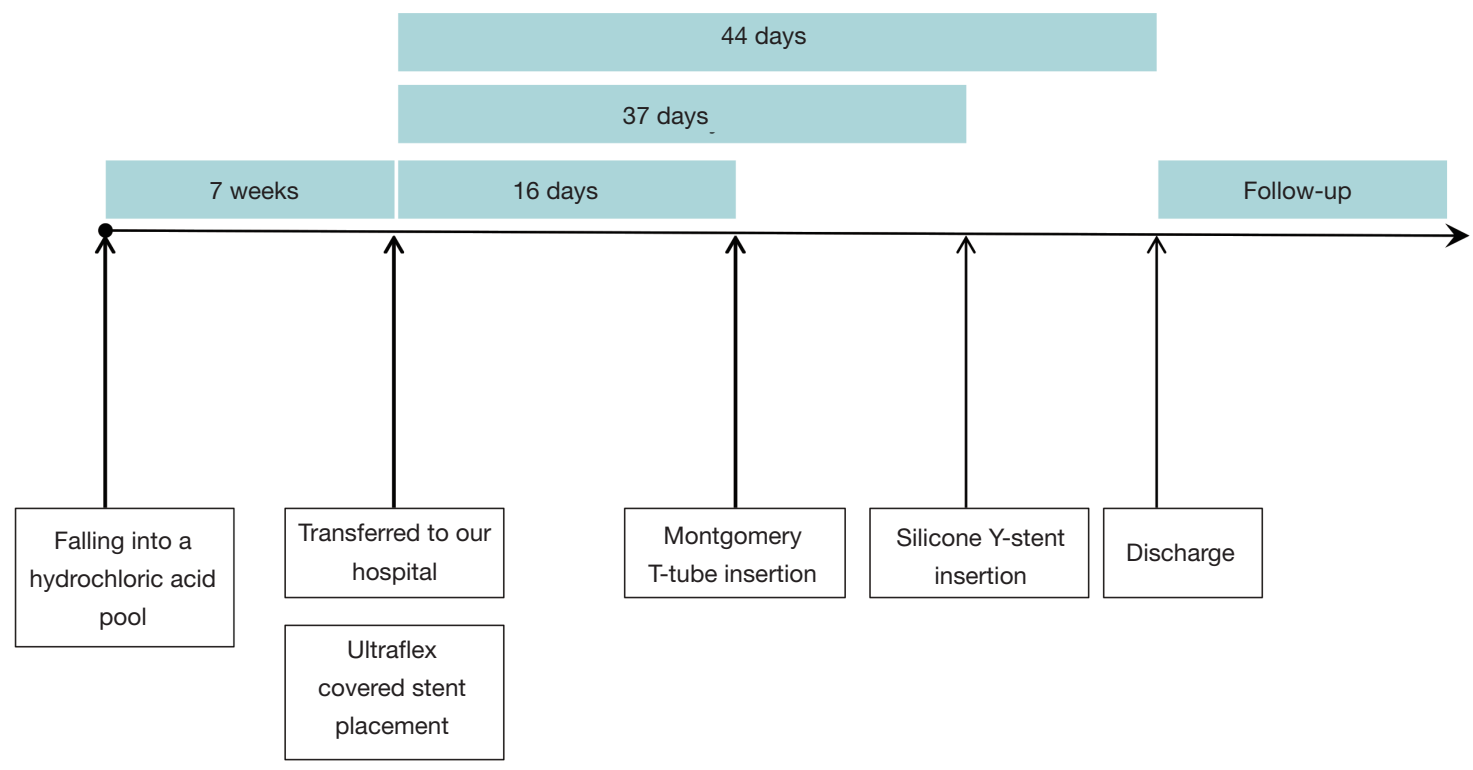

Figure 1 A 45-year-old female experienced dyspnea after falling into a hydrochloric acid pool. Seven weeks later, she was transferred to our hospital and Ultraflex covered stent was inserted subsequently. On the $16^{\text {th }}$ day of admission, a Montgomery T-tube was inserted. On the $37^{\text {th }}$ day of admission, a silicone Y-stent was implanted. She was discharged 44 days after the hospitalization and received a 15-month follow-up.

bronchus. Therapeutic measures contained laryngeal mask ventilation, balloon dilatation and provisional Ultraflex covered stent placement (Figure 1). She was referred for tracheal extubation in 2 days after surgery.

On the $16^{\text {th }}$ day of admission, bronchoscopy revealed that the Ultraflex stent was in position but obviously narrowed during expiration, with obviously collapsed subglottic primary bronchi (Annex 1). The functional Ultraflex stent should not be removed. And a Montgomery T-tube was inserted urgently (Figure 1).

On the $32^{\text {nd }}$ day of admission, a chest computed tomography (CT) and three-dimensional structure reconstruction of trachea showed evident TBM and stenosis in the lower margin of Ultraflex stent. The narrowest lumen diameter was only $2-3 \mathrm{~mm}$. On the $37^{\text {th }}$ day of admission, a silicone Y-stent was implanted (Figure 1). The symptoms remitted obviously. Chest $\mathrm{CT}$ revealed significant improvement in tracheal stenosis (Figure 2).

She was discharged 44 days after the hospitalization with improved breathlessness (score 2 on the modified Borg scale) (Figure 1). Medical orders included atomization treatment, backslapping to help sputum excretion and airway management.

In 50 days after discharge, she scored 1 on modified Borg scale. Lung function showed severe obstructive ventilation dysfunction. And bronchoscopy revealed stents maintained patency with light overlap (Annex 2). In 4 months after discharge, she presented with hoarseness. The symptom disappeared after pruning the upper branch of T-tube. In 5 months after discharge, her lung function showed moderate obstructive ventilation dysfunction. She can participate in daily activities normally without breathlessness (score 0 on the modified Borg scale). In 8 and 15 months after discharge, bronchoscopy viewed a few granulation tissues at the end of stents of bilateral main bronchus respectively (Figure 1). And the granulation was cleaned timely.

All procedures performed in studies involving human participants were in accordance with the ethical standards of the institutional and/or national research committee(s) and with the Helsinki Declaration (as revised in 2013). Written informed consent was obtained from the patient for publication of this case report and any accompanying images.

\section{Comment}

Chemical inhalation injury related to extensive TBM in adults has been rarely reported. The inhalation of hydrochloric acid can seriously damage the upper respiratory tract, and, potentially, severe tracheobronchial stenosis. Bronchoscopy is the gold standard for the diagnosis of airway malacia (2). 

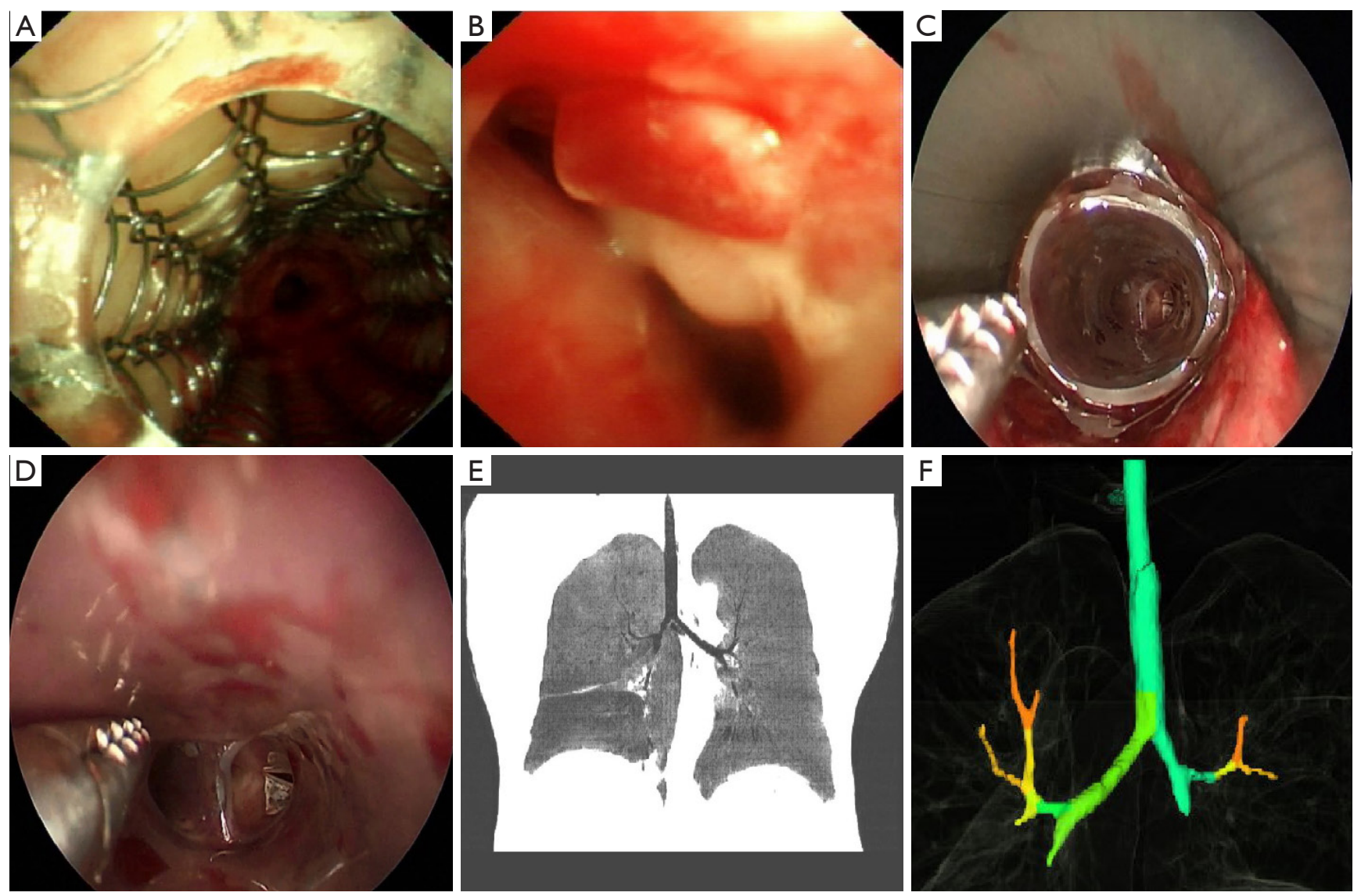

Figure 2 Silicone Y-stent insertion under rigid bronchoscopy. (A) Ultraflex stent was in place; (B) obvious collapse and malacia of carina during expiration; (C,D) silicone Y-stent insertion; (E,F) postoperative chest CT and three-dimensional reconstruction of trachea. CT, computed tomography.

Other diagnostic evaluation includes chest CT with dynamic expiratory imaging, pulmonary function tests.

Airway stents have been applied in management of tracheobronchial pathologies since the second half of the 20th century (3). Common complications included restenosis, stent migration and bad breath. Granuloma formation accounted for $5-44 \%$ of the magnitude of airway restenosis (4).

In this report, the patient developed TBM because of the inhalation of hydrochloric acid. Bronchoscopy indicated the diagnosis of TBM, which revealed tracheal collapse and airway stenosis for more than $80 \%$. The pathogenesis of the patient is believed to be a comprehensive consequence of inflammation and tracheal wall structure changes. The current main management of such severe airway collapse is insertion of airway stents (4). The inhibition of bronchial mucociliary clearance is a common problem associated with stents, which related to bad breath. In this case, the airway malacia appeared and aggravated gradually, stents insertion can relieve dyspnea and correct respiratory failure rapidly. Moreover, granulation was the main complication observed in 15-month follow-up. The supporting function of airway cartilage need to be evaluated, which relating to the removal of airway stents. Considering of long-term complications, we expected to remove the stents in the future.

The presence of TBM or airway collapse should be considered at the onset of dyspnea in patients who unexpectedly breathed in corrosive chemicals. Subsequent close follow-up and tracheal stent insertion is required for achieving the best outcome.

\section{Acknowledgments}

Funding: This work was supported by the National Natural Science Foundation of China (grant number 81770009). 


\section{Footnote}

Reporting Checklist: The authors have completed the CARE reporting checklist. Available at http://dx.doi.org/10.21037/ apm-19-216

Conflicts of Interest: All authors have completed the ICMJE uniform disclosure form (available at http://dx.doi. org/10.21037/apm-19-216). The authors have no conflicts of interest to declare.

Ethical Statement: The authors are accountable for all aspects of the work in ensuring that questions related to the accuracy or integrity of any part of the work are appropriately investigated and resolved. All procedures performed in studies involving human participants were in accordance with the ethical standards of the institutional and/or national research committee(s) and with the Helsinki Declaration (as revised in 2013). Written informed consent was obtained from the patient for publication of this case report and any accompanying images.

Cite this article as: Wang J, Gao L, Tan Qi, Sun W. Multiple stents for tracheobronchomalacia caused by hydrochloric acid inhalation: a case report. Ann Palliat Med 2020;9(5):3593-3596. doi: 10.21037/apm-19-216
Open Access Statement: This is an Open Access article distributed in accordance with the Creative Commons Attribution-NonCommercial-NoDerivs 4.0 International License (CC BY-NC-ND 4.0), which permits the noncommercial replication and distribution of the article with the strict proviso that no changes or edits are made and the original work is properly cited (including links to both the formal publication through the relevant DOI and the license). See: https://creativecommons.org/licenses/by-nc-nd/4.0/.

\section{References}

1. Montgomery WW. T-TUBE tracheal stent. Arch Otolaryngol 1965;82:320-1.

2. Biswas A, Jantz MA, Sriram PS, et al. Tracheobronchomalacia. Dis Mon 2017;63:287-302.

3. Herth FJ, Eberhardt R. Airway stent: what is new and what should be discarded. Curr Opin Pulm Med 2016;22:252-6.

4. Fruchter O, Abed El Raouf B, Rosengarten D, et al. Longterm Outcome of Short Metallic Stents for Lobar Airway Stenosis. J Bronchology Interv Pulmonol 2017;24:211-5. 